\title{
ACTORES PROMOTORES DEL TEMA DE LA VIOLENCIA CONTRA LAS MUJERES EN EL ESPACIO DISCURSIVO PÚBLICO
}

\author{
CARMEN VIVES CASES, MARTA MARTÍN LLAGUNO y Mª JOSÉ FRAU LLINARES \\ Universidad de Alicante
}

\section{INTRODUCCIÓN}

Desde finales de los años ochenta, la investigación sobre violencia contra las mujeres, centrada no tanto en el tema en sí mismo, sino en su desarrollo como cuestión pública y en el análisis de las condiciones de contexto que han potenciado que el tema haya pasado de la "esfera privada» a la "esfera pública», se ha incrementado considerablemente. En este sentido, parece haber un consenso en que los medios de comunicación han jugado un importante papel en la visualización del tema ${ }^{1}$.

Desde su inclusión como tema de investigación, hace ya treinta años, la violencia contra las mujeres ha sido definida, interpretada y abordada desde distintas disciplinas que no sólo han discrepado entre sí en el qué tratar y en el cómo abordarlo, sino que también han diferido en estos aspectos con las personas afectadas por el problema. En este contexto, demarcar el tema que nos ocupa es especialmente complejo ${ }^{2}$. Pese a ello, este estudio se centra exclusivamente en la "violencia contra las mujeres en la pareja», entendida como "cualquier comportamiento dentro de una relación íntima presente o anterior que causa daño físico, psíquico o sexual»³.

1. BERNS, N.: «Degendering the problem and gendering the blame: Political discourse on women and violence", Gender and Society, 15:2 (2001), pp. 262-281.

2. RENZETTI, C.; EDLESON, J. y BERGEN, R.: Sourcebook on violence against women, U.S., California, Sage Publications, 2001.

3. OMS: World Report on violence and Health, Geneva, World Health Organization, 2002: 20, 305, 112 [accedido 5-XI-2003]. Disponible en: http://www5.who.int/violence_injury_prevention/download.cfm?id=0000000582 


\section{EMERGENCIA DEL TEMA LA VIOLENCIA CONTRA LAS MUJERES EN EL ESPACIO DISCURSIVO PÚBLICO}

La violencia contra las mujeres comenzó a hacerse patente en Inglaterra y Estados Unidos a propósito de las reivindicaciones de la legalización de la separación conyugal y el divorcio que lideró la "primera ola del feminismo" (18501950). Sin embargo, no fue hasta mucho después cuando la violencia contra las mujeres entró a formar parte de sus políticas. Así, en las décadas de 1960 y 1970 , los grupos feministas se organizaron en torno a reivindicaciones por el logro de ayudas económicas y sociales para las mujeres víctimas de malos tratos ${ }^{4}$. A partir de estos momentos, comenzaron a darse una serie de acontecimientos en el contexto internacional que fueron claves también para la inclusión de este tema en la agenda política española.

En primer lugar, la Convención de Naciones Unidas para la eliminación de toda forma de discriminación contra la mujer en 1979. Por entonces se dieron en España las primeras campañas de carácter institucional para denunciar la violencia contra las mujeres. En segundo lugar, la III Conferencia Mundial sobre las Mujeres celebrada en Nairobi en 1985. Fue entonces cuando la violencia contra las mujeres emergió como un verdadero problema de la comunidad internacional. En tercer lugar, y por último, la IV Conferencia Mundial sobre las Mujeres (Beijing, 1995) donde se identificó la violencia contra la mujer como un obstáculo para la igualdad, el desarrollo y la paz entre los pueblos que impide que las mujeres disfruten de sus derechos humanos y libertades fundamentales ${ }^{5}$.

A mediados de los años 90, la violencia contra las mujeres comenzó por tanto a consolidarse como un problema legalmente reconocido, que también empezó a captar la atención mediática. Así, en España, se ha afirmado que el tema empezó a aparecer en la prensa de forma diferente, primero, a raíz de la cobertura mediática de las primeras reivindicaciones de los grupos feministas en torno al problema de la violación de mediados de los $80 \mathrm{y}$, posteriormente, cuando las redacciones empezaron a introducir en sus rutinas informativas los primeros momentos de deslegitimación y condena oficial de la violencia contra las mujeres ${ }^{6}$.

Esos acontecimientos no provocaron, sin embargo, un incremento del volumen de noticias sobre el tema como el que se produjo con determinados casos de relevancia social7. Así, en España, se reconoce el impacto mediático de los

4. WALBY, S.: Theorising Patriarchy, Oxford, Blacwell, 1990.

5. MARUGÁN, B. y VEGA, C.: «El cuerpo contra-puesto. Discursos feministas sobre la violencia contra las mujeres», VIII Congreso de Sociología, Salamanca, 2001 [accedido 25-VII-2003]. Disponible en: http://www.sindominio.net/Karakola/textos.htm

6. Fagonga, C.: La violencia en los medios de comunicación. Maltrato en la pareja y agresión sexuada, Madrid, Dirección General de la Mujer, 1999.

7. Consalvo, M.: «3 Shot Dead in Courthouse: Examining News Coverage of Domestic Violence and Mail-order Brides», Women's Studies in Communication, 21:2 (1998), pp. 188-211. MAXWELL, K.; HuxFord, J.; BORUM, C. y HornIK, R.: «Covering Domestic Violence: How the O.J. Simpson case shaped reporting of Domestic Violence in the news media", Journalism and Mass Communication 
casos de Lorena Bobbit de 1993, de las niñas de Alcàsser de 1995 y de Ana Orantes de finales de $1997^{\circ}$.

En el contexto descrito, se plantea este estudio con el objetivo de identificar los acontecimientos y actores que han contribuido, entre 1997 y 2001, al mantenimiento del tema en los periódicos españoles y en el orden del día del Congreso de Diputados y del Senado.

\section{METODOLOGÍA}

Se llevó a cabo un estudio cuantitativo basado en dos tipos de fuentes de información. Por un lado, fueron seleccionadas todas las noticias publicadas desde enero de 1997 hasta diciembre de 2001 en las ediciones digitales e impresas de El País, El Mundo, y el ABC (los tres periódicos de información general con mayor difusión media en el ámbito nacional, según la OJD) y el diario Información de Alicante (el periódico de mayor tirada en la provincia de Alicante), cuyo título o antetítulo contuviese uno o varios de los siguientes términos: violencia doméstica, maltrato/ malos tratos, violencia contra la mujer, violencia de género y muerte. Se reunió un total de 1491 noticias.

Por otro lado, se realizó una búsqueda en el Diario de Sesiones del Congreso de Diputados y del Senado para localizar todas las iniciativas legislativas, propuestas de ley orgánica, propuestas no de ley, informes, ponencias, enmiendas y preguntas al Gobierno sobre el tema objeto de estudio, recogidas en el Diario de Sesiones del Congreso de Diputados y del Senado. Fueron analizadas todas las actas de las sesiones desarrolladas desde el 1 de enero de 1997 hasta el 31 de diciembre 2001. Se reunió un total de 174 actas de sesiones parlamentarias en las que el tema objeto de estudio apareció mencionado o incluido entre los temas del orden del día.

Se realizó un análisis de contenido cuantitativo, como se viene realizando en los trabajos de la investigación sobre la agenda ${ }^{9}$ y en algunos sobre la cobertura

Quarterly, 77: 2 (2000), pp. 258-272. BULLOCK, C. y CUBERT, J.: "Coverage of domestic violence fatalities by newspapers in Washington State», Journal of Interpersonal Violence, 17:5 (2002), pp. 475-499.

8. ALBERDI, I. y MATAS, N.: La violencia doméstica. Informe sobre los malos tratos a mujeres en España, Barcelona, Fundación «La Caixa», 2002. Colección de Estudios Sociales 10 [accedido 3-XI-2003]. Disponible en: http://www.estudios.lacaixa.comunicacions.com/webes/wpp0pdfp.nsf/vico/ es10_esp.pdf/\$file/es10_esp.pdf

9. RIFFE, D. y FreITAG, A.: "A content analysis of content analyses: Twenty-five years of yournalism quarterly», Journalism mass communication, 74:4 (1997), pp. 873-882. WALDMAN, P. y DEVITT, J.: «Newspaper photographs and the 1996 presidential election: The question of bias», Journalism mass communication, 75:2 (1998), pp. 302-311. SHAW, D. y SPARROW, B.: «From the inner ring out: News congruence, cue-taking and campaign coverage», Politic Research Quarterly, 52:2 (1999), pp. 323-351. BANWART, M.; BYSTROM, D. y ROBERTSON, T.: "From the primary to the general election. A comparative analysis of candidate media coverage in mixed-gender 2000 races for governor and US Senate», American Behaviour Science, 46:5 (2003), pp. 658-676. GoULD, M.; JAMIESON, P. y ROMER, D.: "Media contagion and suicide among the young», American Behaviour Science, 46:9, pp. 1269-1284. 
periodística del tema objeto de estudio ${ }^{10}$. Este análisis permite identificar en el texto una serie de variables, que van desde la posición en la página hasta la postura sobre un tema ${ }^{11}$. Para ello se elaboró un código de análisis donde se establecieron variables referentes a tres categorías relevantes para esta investigación:

- Clasificación periodística (fecha de publicación, año, mes, día de la semana, periódico y sección).

- Promotores: autor/es en la firma, sexo de los autores e identificación de las principales fuentes informativas en el cuerpo de la noticia por sexo, por características personales o profesionales -allegados o vecinos, personal judicial, político, médico, agente social- o por tipo de colectivo que representa-organizaciones no gubernamentales, instituciones públicas-.

- Tipo de información divulgada sobre el tema: mención de factores de riesgo, sucesos (agresiones, violaciones, muertes) y medidas (de coerción o castigo a los agresores, de asistencia a las víctimas, de prevención del problema).

- Tratamiento específico del problema de los malos tratos: tipo de violencia noticiada (física/ psíquica, sólo física, sólo psíquica, sexual), características nombradas en torno a las víctimas (sexo y edad) y los agresores (sexo y edad).

Por su parte, con respecto a las actas de las sesiones parlamentarias, al igual que en el caso de las noticias sobre malos tratos, se realizó un análisis de contenido de los textos (cuantitativo) guiado por un código de análisis en el que se tuvieron en cuenta los siguientes aspectos:

- Fecha del acta (año, mes y día).

- Identificación del órgano en el que se celebró la sesión (Pleno del Congreso de Diputados, Pleno del Senado o Comisiones -de las relaciones con el Defensor del Pueblo, de Justicia e Interior, de Política Social y Empleo, de Presupuestos, especiales para problemas con el medio rural, del control parlamentario de RTVE, Constitucional, de Sanidad y Consumo, de los Derechos de la Mujer).

- Identificación de los actores promotores de intervenciones parlamentarias (Grupo Popular, Socialista, de Izquierda Unida, Catalán, Canario, Vasco, Mixto, otros grupos parlamentarios, Defensor del Pueblo, Comisión de investigación sobre malos tratos, Instituto de la Mujer, Ministerio de Trabajo y Asuntos Sociales, Ministerio de Justicia, Ministerio del Interior, otros ministerios, Gobierno, Expertos de violencia contra las mujeres, Secretaría de Asuntos Sociales, otras secretarias, otros interlocutores) y sexo de los protagonistas de las intervenciones.

10. STONE, S.: "Getting the message out: feminist, the press and violence against women", The Canadian Review of Sociology and Antropology, 30:3 (2003), pp. 377-400. PRASAD, D.: "Dowry-related violence: A content analysis of news in selected newspapers", Journal of Comparative Family Studies, 25:1 (1994), pp. 71-86. Bullock, C. y Cubert, J.: Op. cit. MAXWell, K.; HuXford, J.; BORUM, C. y HORNIK, R.: Op. cit.

11. García-Ferrando, M.; Ibáñez, J. y Alvira, F.: El análisis de la realidad social. Métodos y Técnicas de Investigación social, Madrid, Alianza, 2000. 
- Motivo de la Sesión (proposición de ley o no de ley, preguntas, contestaciones, ponencias o informes, votaciones).

- Carácter de las medidas e iniciativas desarrolladas (de prevención, asistencia, investigación y/o coerción).

Una vez codificada la información resultante, se calcularon básicamente frecuencias, porcentajes y se realizaron comparaciones de porcentajes basadas en las pruebas $\mathrm{X}^{2}$. También se calcularon correlaciones bivariadas basadas en el coeficiente r de Pearson. Para estos cálculos, se utilizó el programa SPSS-12.

\section{ANÁLISIS DE RESULTADOS}

\subsection{Visibilidad del tema en las noticias de prensa}

Tal y como ya se evidenció en la revisión bibliográfica, a finales de los años 90 , un hecho concreto supuso un salto cuantitativo y cualitativo en la cobertura periodística del tema: el caso de Ana Orantes. Cinco años después, puede decirse que la cobertura periodística de la violencia contra las mujeres continuó creciendo, aunque no de forma homogénea. [ver figura 1]

Aunque resulta especialmente llamativo el clímax de informaciones entre diciembre del 97 y enero del 98 ( $n=92)$, el descenso observado en el número de noticias publicadas durante los meses siguientes a este caso permite, en principio, afirmar que el tema no logró consolidarse en la agenda periodística española hasta pasada la mayor parte del año 1998 (enero: 92 noticias -24,7\% del total de 1998-; febrero: 27 noticias -7,2\%) [figura 1].

En octubre de 1998, se produce otro clímax de atención, provocado inicialmente por la irrupción de «medidas» pero aderezado ipso facto por una lluvia

Figura 1

Distribución mensual del número de noticias de violencia contra las mujeres en El País, El Mundo, ABC e Información de Alicante. Evolución 1997-2001

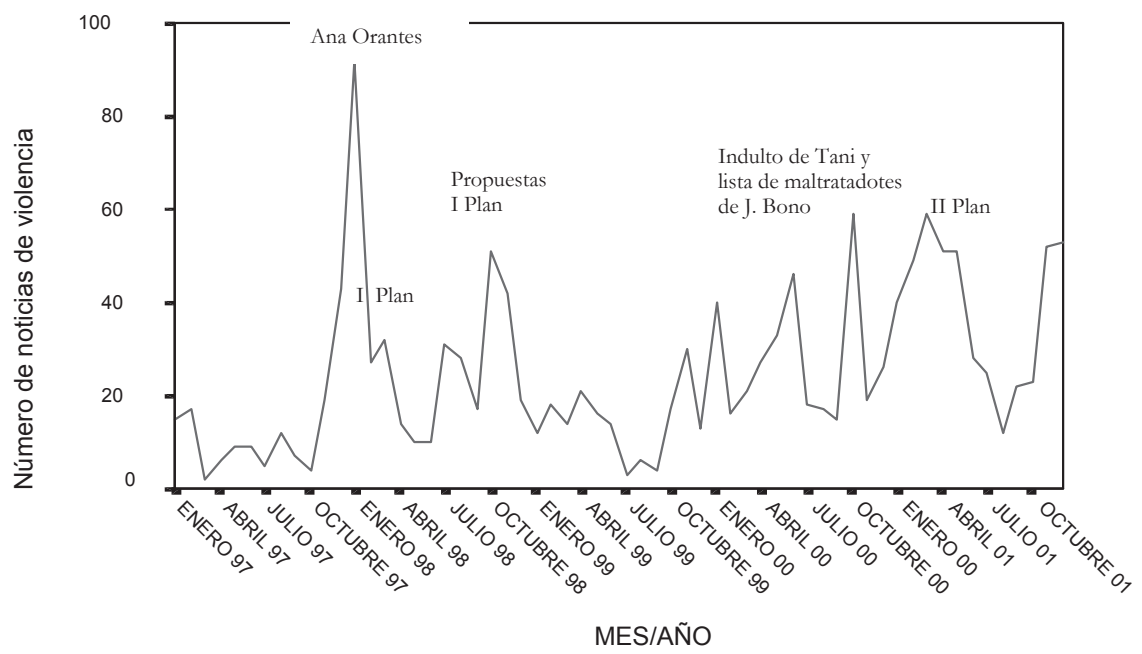


de sucesos. Inicialmente, en este mes, encontramos un aluvión de informaciones sobre las estrategias propuestas por las instituciones responsables de la ejecución del I Plan oficial contra la violencia hacia las mujeres, aprobado por el consejo de ministros en abril de este mismo año -Fiscalía General, policía o Ministerios de Interior y Asuntos Sociales-. Pero, estas informaciones se ven salpicadas por noticias centradas en casos de violencia contra las mujeres. No se trata de "historias de vida» concretas que aparecen sostenidamente en la prensa, sino de una gran variedad de experiencias de las que sólo se informa del momento en que se produce la agresión. Esta lluvia de pequeñas informaciones parece ubicarse intencionadamente en los medios de comunicación como presión o justificación del cumplimiento del mencionado I Plan oficial.

No es hasta dos años después, en octubre de 2000, cuando se detecta otro pico de informaciones provocado de nuevo por dos sucesos periodísticos que incitan a la cobertura de medidas [ver figura 1]. A principios de este mes, surge el caso de la propuesta de indulto de "Tani», una mujer sentenciada a prisión por haber sido la responsable de la muerte de su marido y agresor. El indulto de "Tani» da lugar a una oleada de informaciones sobre otros casos de características similares y hace también aparecer opiniones diversas sobre la posibilidad de conceder el indulto bajo este tipo de condiciones. Además, a mediados de octubre de 2000, la prensa cubre también otra controvertida cuestión, que da lugar a opiniones contrapuestas en los diarios: la propuesta del presidente de la Comunidad de Castilla la Mancha, José Bono, de hacer pública una lista con los nombres de hombres que han sido juzgados y penalizados por maltratar a mujeres. Este proyecto provoca no sólo la aparición de opiniones y manifestaciones desde organismos gubernamentales y no gubernamentales, sino también de revisión en los medios de la eficacia de las distintas medidas de control sobre los agresores y la denuncia pública de los casos en los que resulta evidente la limitada capacidad de protección oficial de las víctimas de malos tratos.

La propuesta del Presidente de la Comunidad de Castilla la Mancha continúa siendo eje de debate en los periódicos hasta bien entrado el año 2001. En la primera mitad de este año, se observa que sigue creciendo también la cobertura periodística de opiniones y posiciones adoptadas por el Consejo General del Poder Judicial respecto a la limitada protección que las propuestas de intervención vigentes garantizan a las mujeres que deciden denunciar. Consecuentemente, se observa en la figura 1 un cuarto clímax de noticias sobre el tema en marzo de 2001, promovido, principalmente, por una cobertura periodística de iniciativas para dar solución a la limitada protección de las mujeres, como, por ejemplo, la protagonizada por el propio Presidente de la Comunidad de Castilla la Mancha de «hacer públicos los presupuestos dirigidos a luchar contra la violencia doméstica» (El Mundo, 13 de marzo de 2001).

Por último, también es necesario reflexionar sobre una «crisis» que se produce en la cobertura periodística durante el verano de 1999 [ver figura 1], cuando prácticamente los medios dejan de hablar de la violencia contra las mujeres. La explicación más plausible para este descenso tan dramático puede ser que los medios de comunicación tienden a publicar menos noticias cuando el ejecutivo 
adopta una posición definida con respecto a los problemas, mientras aumentan el volumen de informaciones sobre estos cuando la posición de los Gobiernos ante los problemas no está definida ${ }^{12}$. Así, cabe la posibilidad de que, en 1999, la cobertura periodística general del tema disminuyera porque la presentación, en abril de 1998, del I Plan oficial contra la violencia hacia las mujeres puso de manifiesto una clara posición del Gobierno español contra este problema.

\subsection{Evolución de las sesiones parlamentarias de violencia contra las mujeres}

En general, podría decirse que en función de lo que se observa en la figura 2 , la evolución de la presencia de la violencia contra las mujeres en las sesiones parlamentarias presenta una tendencia claramente inestable y no necesariamente creciente.

Se observa en la figura 2 que, en los años en los que tuvieron lugar las discusiones sobre los contenidos de los dos planes oficiales contra la violencia hacia las mujeres -1998 y 2001-, la frecuencia de aparición del tema en las sesiones parlamentarias es mayor. Además, en estos mismos años, también se incrementa la presencia del tema en el orden del día de las sesiones parlamentarias. Más específicamente, el tema aparece en el orden del día del 64\% de las sesiones de 1998 y del $72 \%$ de las de 2001.

La recuperación observada en el año 2000, tras la crisis de 1999 [ver figura 2], podría estar relacionada con el hecho de que se trata de un año intermedio entre la aprobación del I Plan y II Plan contra la violencia hacia las mujeres, en el que los representantes políticos comienzan a plantear cuestiones al Gobierno sobre el desarrollo del primero y los términos en los que se formularán las medidas contempladas en el segundo.

Por otro lado, puede plantearse la posibilidad de que los incrementos en la actividad parlamentaria se relacionen con los incrementos observados en la

\section{Figura 2}

Distribución porcentual por años de las sesiones parlamentarias de violencia contra las mujeres. 1997-2001

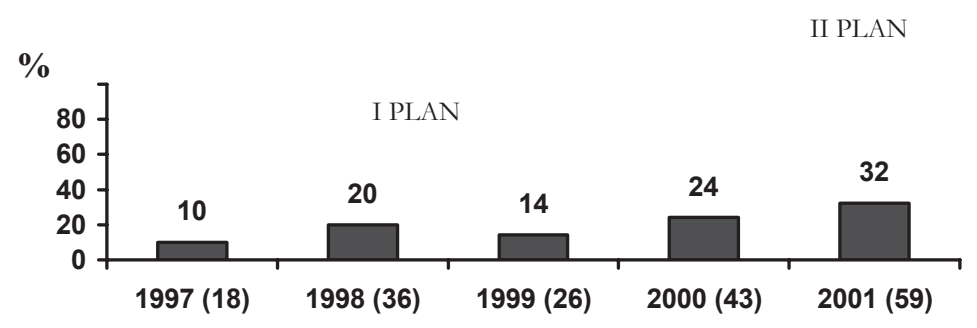

12. ECKSTEIN, H.: "Case Study and Theory in Political Science», en F. Greenstein y N. Polsby (eds.): Handbook of Political Science Strategies of Inquire, MA, Addison-Wesley, 1975, pp. 79-137. 
cobertura periodística. La realización de las pruebas estadísticas nos permiten confirmar la hipótesis de que los incrementos en la cobertura influyeron, de algún modo, en los incrementos de la actividad parlamentaria $(r=0,355 ; p=0,005)$ en el mismo mes en el que se publican las noticias, y continúan haciéndolo con mayor intensidad un mes después de su aparición en los periódicos $(r=0,545$; $p=0,001)$. Es decir, parece existir cierta relación entre el hecho de que la cuestión sea tema de noticia y sea tratada en las sesiones parlamentarias.

\subsection{Actores promotores de noticias y sesiones parlamentarias sobre el tema}

En las noticias de violencia contra las mujeres publicadas entre 1997 y 2001 -en total 1491- destacaron como autores los periodistas (52\%) y las agencias de noticias $(25 \%)$. El porcentaje restante fue mayoritariamente de noticias sin firma, pero basadas en la información proporcionada por agencias de noticias (23\%). Cabe destacar no obstante que, con el paso del tiempo, las noticias firmadas por periodistas fueron cediendo terreno a las noticias de agencias. Así, más de la mitad de las 775 noticias firmadas por periodistas se publicaron entre $2000(26 \%)$ y $2001(32 \%)$.

En la mayoría de las noticias firmadas aparecieron como autoras mujeres periodistas $(55 \%) \mathrm{y}$, en estas noticias firmadas por ellas se presentaron como fuentes principales el doble de mujeres que en las firmadas por hombres $(p=0,02)$. Esta asociación es importante, teniendo en cuenta que la voz de las mujeres en las noticias de violencia no fue en general tan visible como era de esperar (fuentes principales sobre total noticias -1491-; hombres: 51\%; mujeres: $49 \%$ ).

La tendencia de la distribución por sexo de las fuentes principales sólo se invirtió en 1999, cuando las mujeres hicieron más declaraciones sobre la violencia contra ellas en las noticias de prensa que los hombres [ver figura 3]. Parece que, nuevamente, ellas desarrollaron un papel clave en el mantenimiento del tema

Figura 3

Fuentes principales de las noticias de violencia contra las mujeres por sexo.

1997-2001. $\mathrm{N}=559$

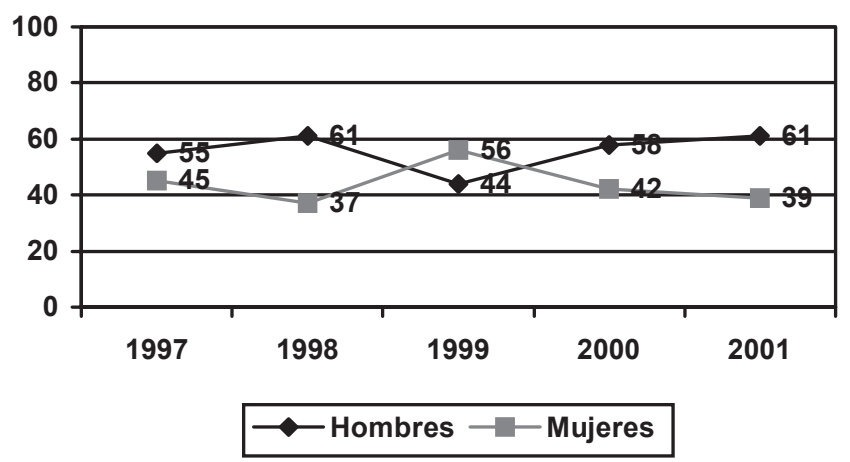


Actores promotores del tema de la violencia contra las mujeres en el espacio...

Tabla 1

Distribución por sexo y sector profesional-personal de las fuentes principales. Noticias de violencia contra las mujeres. 1997-2001

\begin{tabular}{|c|c|c|c|c|c|}
\hline \multirow[b]{3}{*}{ Sector profesional-personal } & \multicolumn{4}{|c|}{ Fuente principal } & \multirow{3}{*}{ Total } \\
\hline & \multicolumn{2}{|c|}{ Hombre } & \multicolumn{2}{|c|}{ Mujer } & \\
\hline & $\mathbf{N}$ & $\%$ & $\mathbf{N}$ & $\%$ & \\
\hline Sanitario & 6 & 33,3 & 12 & 66,7 & 18 \\
\hline Político & 100 & 48,8 & 105 & 51,2 & 205 \\
\hline Grupos feministas & 4 & 4,3 & 89 & 95,7 & 93 \\
\hline Jurídico/Judicial & 135 & 80,8 & 32 & 19,2 & 167 \\
\hline Familiar/Vecindario/Amistad & 8 & 72,7 & 3 & 27,3 & 11 \\
\hline Periodístico & 19 & 41,3 & 27 & 58,7 & 46 \\
\hline Sin especificar & 11 & 57,9 & 8 & 42,1 & 19 \\
\hline Total & 283 & 50,6 & 276 & 49,4 & 559 \\
\hline
\end{tabular}

$p<0,001$

en las páginas de los periódicos españoles. Por su parte, los hombres destacaron como fuentes principales de las noticias, sobre todo, en los años en los que los periódicos colmaron sus esfuerzos por difundir informaciones sobre lo acordado en los planes contra la violencia hacia las mujeres. Así, su participación en calidad de fuentes principales destacó en 1998 y 2001, los periodos de mayor institucionalización del tema [ver figura 3].

La clasificación de las fuentes principales en función de la institución a la que representan permite analizar con mayor detenimiento la distribución de las fuentes por sexo. Concretamente, se observó que la voz de las mujeres políticas y feministas fue utilizada en gran medida para fundamentar la información difundida por la prensa durante el lustro analizado. Los hombres, por su parte, fueron fuente principal de las noticias, sobre todo, en calidad de jueces, fiscales o altos cargos del poder judicial [ver tabla 1].

En función de la distribución observada en la tabla 1 por sectores profesionales y sexo de las fuentes informativas principales, puede decirse que, en algunos casos, el espacio impreso de los periódicos se ve condicionado por la mayor y menor presencia de mujeres en determinados ámbitos. Así, la menor presencia de mujeres que se observa en la tabla en el sector jurídico-judicial podría estar condicionada por la también minoritaria asunción de puestos de mayor responsabilidad en dicho ámbito por parte de las mujeres.

En los años de estudio, las diferencias más notables se dieron en los momentos de máxima difusión sobre el contenido de los planes contra la violencia hacia las mujeres $(p<0,001)$. Así, la proporción de noticias en las que hombres políticos aparecen en calidad de fuentes principales se equiparó en 1998 (hombres: 50\%, mujeres: $50 \%$ ) y se acrecentó en 2001(hombres: 58\%, mujeres: $42 \%$ ). Sin embargo, en los años previos e intermedios de estos planes oficiales, las noticias rescataron con mayor frecuencia las declaraciones de las representantes de la Comisión Mixta de Derechos de la Mujer y otras comisiones encargadas del 
tema en el Parlamento (1997: Hombres 47\%, Mujeres 53\%; 1999: Hombres $34 \%$, Mujeres 66\% y; 2000: Hombres 48\%, Mujeres 52\%). En consecuencia, puede argumentarse que la menor representación de mujeres en los cargos del Gobierno y de gestión se pone de manifiesto en las noticias de violencia contra las mujeres en los momentos de mayor institucionalización del tema.

Por su parte, a lo largo de las 174 sesiones en las que el tema apareció en diversos órganos parlamentarios, intervinieron diferentes partidos políticos. En 1997, en el que el tema fue abordado, sobre todo, a propósito de otras cuestiones - principalmente, con relación a los contenidos del «Proyecto Mujer» de la Unión Europea, los contenidos del III Plan de Igualdad de Oportunidades entre mujeres y hombres, y el debate sobre la ampliación de supuestos sobre la Interrupción Voluntaria del Embarazo-, intervinieron fundamentalmente representantes del Grupo Parlamentario Socialista (60\% sobre las 15 sesiones localizadas en este año), Izquierda Unida (20\%) y Grupo Parlamentario Mixto $(13 \%)$.

En 1998, la violencia contra las mujeres fue introducida en los debates parlamentarios por una mayor variedad de interlocutores. Mientras que en periodo inaugural de la actividad parlamentaria de este año las intervenciones producidas (total 9) fueron exclusivamente realizadas por el Grupo Parlamentario Socialista (89\%) e Izquierda Unida (11\%), es decir, intervenciones de los partidos de la oposición; en los meses posteriores a la presentación de este I Plan, se dio la incorporación de más grupos parlamentarios -entre ellos, el Popular- en esta actividad promotora de la presencia del tema en el Parlamento. Estos resultados sugieren que posiblemente el problema empezó a ser integrado también en las agendas de mayor diversidad de partidos políticos e instituciones públicas.

En 1999, aunque en los debates parlamentarios intervinieron una gran variedad de interlocutores, la presión de los partidos de la oposición disminuyó en intensidad. De hecho, el liderazgo en este caso pasó a manos del Grupo Mixto $(22 \%)$ que, en ese momento, tenía menor representación en el Parlamento. Posteriormente, en el 2000, se incrementaron las preguntas al gobierno -el $21 \%$ de las intervenciones fueron preguntas y el $46 \%$ fueron peticiones de comparecencias para informar- con respecto a los años anteriores. Estas preguntas fueron formuladas fundamentalmente por socialistas (43\%), como también fueron hechas mayoritariamente por ellos las peticiones de comparecencias a miembros del Gobierno (35\%). Uno de los motivos principales por los que se intensificaron los debates sobre el tema fue la propuesta de elaboración del protocolo de atención sanitaria a las víctimas de malos tratos, incluido entre los contenidos del I Plan oficial contra la violencia hacia las mujeres.

Por último, cabe destacar la intervención del líder del Grupo Parlamentario Socialista en la sesión del Pleno del Congreso de Diputados de 7 de marzo de 2001, en la que realizó una pregunta al Gobierno sobre los resultados del I Plan contra la violencia hacia las mujeres. A partir de esta pregunta y hasta la presentación del II Plan contra la violencia hacia las mujeres, los debates en el Parlamento se centraron fundamentalmente en la necesidad de evaluar los resultados del I Plan. Mientras que los socialistas formulaban preguntas en torno a 
Actores promotores del tema de la violencia contra las mujeres en el espacio...

Tabla 2

Distribución porcentual de los actores políticos por sexo. Interlocutores en las sesiones parlamentarias. 1997-2001

\begin{tabular}{|l|c|c|c|}
\hline & Políticos & Políticas & Total Sesiones \\
\hline 1997 & $20 \%$ & $80 \%$ & 15 \\
\hline 1998 & $29 \%$ & $71 \%$ & 35 \\
\hline 1999 & $63 \%$ & $37 \%$ & 24 \\
\hline 2000 & $40 \%$ & $60 \%$ & 44 \\
\hline 2001 & $39 \%$ & $61 \%$ & 56 \\
\hline Total Sesiones & 67 & 107 & 174 \\
\hline
\end{tabular}

$\mathrm{p}<0,001$

los resultados obtenidos por el I Plan contra la violencia hacia las mujeres (40\% de las preguntas), expertos sobre el tema procedentes de diferentes ámbitos comparecieron en la ponencia de estudio para la erradicación de la violencia doméstica. Las intervenciones realizadas en esta nueva cámara no sólo supusieron un incremento de la frecuencia de tratamiento del tema en el Parlamento y de su aparición en el orden del día de las sesiones parlamentarias, sino que también contribuyeron a la incorporación de mayor variedad de interlocutores. Así, las intervenciones de estos expertos colmó el discurso parlamentario de ese año (40\%).

Una vez descrita la actividad parlamentaria en función de los grupos que la promovieron, merece la pena finalizar con un plano más centrado en el individuo. Concretamente, analizando por sexo los representantes de los grupos parlamentarios y demás colectivos anteriormente mencionados. En general, la promoción del debate sobre el tema fue protagonizada por mujeres $(60 \%)$. De estos años, cabe destacar que, en 1999 -año en el que la participación de las políticas como fuentes principales de las noticias fue clave-, los políticos fueron los principales artífices de las intervenciones parlamentarias [ver tabla 2].

Para la interpretación de este resultado cabe tener en cuenta que, en 1999, disminuyó las intervenciones del Grupo Parlamentario Socialista, protagonizadas, en su mayoría, por diputadas y senadoras. La políticas socialistas aportaron el $42 \%$ de intervenciones en general y el $73 \%$ de las realizadas por su grupo en los cinco años analizados. Por tanto, puede decirse que, al disminuir las contribuciones de este grupo, también disminuyó la proporción general de mujeres interlocutoras en las sesiones parlamentarias. Además, ya se dijo que, en 1999, el Grupo Parlamentario Mixto asumió la mayor proporción de intervenciones. Las representantes de este grupo sólo fueron un $4 \%$ del total de interlocutoras e hicieron menos intervenciones que sus compañeros de grupo (Total de intervenciones del Grupo Parlamentario Mixto: 10; mujeres: 4 y hombres: 6). También es importante recordar que, en este mismo año1999, fue menor la proporción de sesiones en las que se realizaron preguntas a los miembros del 
Gobierno en comparación con los otros años. Este tipo de intervenciones fueron, fundamentalmente, realizadas por mujeres $(70 \%)$.

Al margen de estas explicaciones sobre la disminución de la participación de las mujeres en la actividad parlamentaria, cabe recordar, por último, que durante esta época ellas continuaron promoviendo el debate sobre el tema en el espacio discursivo mediático en calidad de fuentes principales de noticias de prensa.

\section{CONCLUSIONES}

- La violencia contra las mujeres en España fue inicialmente promovida en el espacio discursivo público por los grupos feministas españoles. En este sentido, cabe destacar las condiciones del contexto socio-político internacional y nacional y la lucha que libraron de la clandestinidad, facilitando estos grupos así la integración de sus reivindicaciones en la agenda política española.

- Estos grupos de presión iniciales fueron, sin embargo, extendiéndose y diversificándose a lo largo del tiempo. Así, a la acción pionera de las feministas cabe añadir la actividad desarrollada y que continúan ejerciendo algunas mujeres pertenecientes a otros ámbitos, como el político y el periodístico.

- La mortalidad por violencia contra las mujeres reflejada en la prensa se presenta como el elemento detonante del reconocimiento público de este problema. En este sentido, cabe destacar el impacto de casos índice de relevancia social -como el de la señora Ana Orantes- sobre las instituciones mediática y política. En este sentido, la iniciativa de una mujer maltratada de romper el silencio ante los medios de comunicación es comúnmente identificada como el punto de partida de la construcción del problema de la violencia contra las mujeres en España. Puede decirse que la cobertura mediática de este caso puso en evidencia la necesidad de que las instituciones públicas y el Gobierno tomaran una postura oficial con respecto al tema.

- La prensa, al igual que es reflejo de la evolución de la incidencia de la violencia contra las mujeres -con relación a las denuncias y las muertes- y de la actividad política que se desarrolla en torno a esta cuestión, también lo es de la desigualdad en la distribución de cargos con responsabilidad existente en las instituciones políticas. Por tanto, se corroboran las funciones de los medios de comunicación de explorar la realidad y detectar las anomalías o, tal y como plantearon los entrevistados, de difundir información y hacer visibles los problemas.

- El tratamiento del tema en las sesiones parlamentarias durante los cinco años estudiados es, con mayor frecuencia, promovido por partidos en la oposición. Así, la presencia del tema en el orden de día de las sesiones depende todavía de la agenda de estos partidos y, sobre todo, de las mujeres pertenecientes a estos. 